\title{
Research on the Application of Tujia Brocade Pattern in Modern Cultural and Creative Design*
}

\author{
Hong Nie \\ School of Art and Design \\ Wuhan University of Science and Technology \\ Wuhan, China 430065
}

\author{
Shulin Zhang \\ School of Art and Design \\ Wuhan University of Science and Technology \\ Wuhan, China 430065
}

\begin{abstract}
Tujia brocade is the handmade handicraft handed down from generation to generation among Tujia women, with strong local characteristics and is an important manifestation of Tujia spiritual wealth. Taking the Tujia brocade pattern as the research object, this paper explores how to integrate the Tujia brocade pattern into the cultural and creative design in the modern visual aesthetic way by analyzing the integration and coincidence of the Tujia brocade pattern and the modern cultural and creative design, and then proposed that the design elements and colors of brocade can be refined and flattened artistically, and applied to modern design and development of related Tujia cultural and creative products, so as to improve the visual communication and cultural transmission of Tujia brocade pattern, promote its sound development, and thereby strengthening the research and inheritance of Tujia brocade pattern.
\end{abstract}

Keywords-Tujia brocade pattern; cultural and creative design; flattening; deconstruction

\section{INTRODUCTION}

Under the economic globalization, people pay even more attention to the living inheritance and protection of regional culture. How to integrate the cultural genes accumulated over thousands of years in Tujia brocade with the development trend in the new era and transmit them in a modern way has become a new topic. The author believes that tradition and modernity, handicraft and science and technology are not contradictory. We should keep pace with the development of the times, integrate new science and technology into traditional culture perfectly, and transform it into a new culture and art with typical Chinese characteristics. On the basis of guaranteeing the pattern, meaning and charm of Tujia brocade, it is of practical significance to incorporate modern design concepts and study the innovative application of Tujia brocade pattern in modern cultural and creative design from the perspective of modern aesthetics, so as to inject new vitality into traditional culture and promote its good development.

*Fund Project: This paper is a phased achievement of the provincial teaching research project "Research on the Practical Teaching Model of Visual Communication Design Major from the Perspective of Community Service" in Hubei Province in 2017 (Project No. 2017245); phased achievement of the general project of humanities and social science research of Hubei Education Department in 2018 "Urban Color Culture Research Based on GIS Platform: A Case Study of Wuhan Central District" (Project No 18Y029).

\section{ARTISTIC CHARACTERISTICS AND CONTEXT}

Tujia brocade was called "Xibu" in ancient times. It has a history of thousands of years. In Ming and Qing Dynasties, it was also called "Banbu" and "Tujin". In traditional brocade, the main materials used are silk thread and cotton thread, and the weft threads used are mostly wool fiber thread, such as yarn and acrylic fiber, etc. The unique artistic beauty of traditional Tujia brocade is the result of making full use of tools and materials by Tujia people and the artists' superb artistic attainments. Complete handicraft operation distinguishes folk handicraft products from industrial and cultural products. The folk craft represented by Tujia brocade is famous for its unique making skills.

Tujia brocade can be divided according to the two weaving techniques: plain weave and twill weave. The yarns with different colors are picked out from the weft upward. When picking up, the residual weft is floated on the front and the pattern is formed on the back. Fine warp and thick weft, with weft gram warp, the pattern part only shows color weft, no basic warp and dark weft. There is a gap between the color weft boundary and the ground warp and dark weft of the pattern, which produces a stereoscopic feeling similar to that of carving and hollowing out. In the specific weaving process, although it is made according to the procedure, the procedures of counting yarns and color weft are usually carried out according to ones experience and feeling, so even the same pattern may have some changes. The unique artistic beauty of traditional Tujia brocade is the result of making full use of tools and materials by Tujia people and the artists' superb artistic attainments. Complete handicraft operation distinguishes folk handicraft products from industrial and cultural products. The folk craft represented by Tujia brocade is famous for its unique making skills.

\section{A. Pattern}

1) Basic frame and composition characteristics: From the perspective of pattern composition, Tujia brocade is of typical plane composition. Each kind of Tujia brocade pattern is arranged and combined by three elements: point, line and surface, with rigorous balanced symmetry characteristics both vertically and horizontally. Traditional Tujia brocade patterns usually take the geometric shapes like diamond, square, triangle or complex polygon as the basic frame, and then fill 
the appropriate pattern in each unit that is divided. The filling pattern is also generally symmetrical, as shown in "Fig. 1".

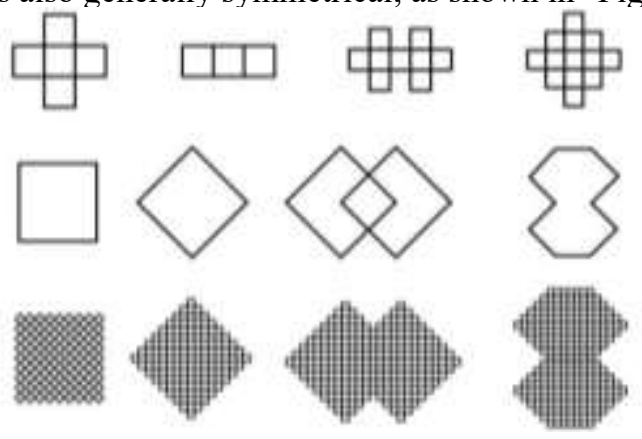

Fig. 1. The basic frame of Tujia brocade.

Traditional Tujia brocade patterns can be divided into three parts: upper, middle and lower. The upper and lower parts of the brocade are called "Dangtou" by the Tujia people. Generally, the upper and lower parts of the brocade are in the same patterns, which are distributed in horizontal strips and presented in two-way continuous form. The middle is the main part of the brocade, and the patterns are varied, mostly in vertical strip style. The main pattern of this part is usually symmetrical and continuous, and the auxiliary pattern is used to connect or fill the gap between the main patterns, thus not only creates an atmosphere of unity and opposites between complexity and simplicity, sparseness and density, but also highlights the variety and gorgeousness of Tujia brocade patterns. Under the unified brocade frame, the rich and varied patterns of the main part better reflect the decorative nature of Tujia brocade, as shown in "Fig. 2".

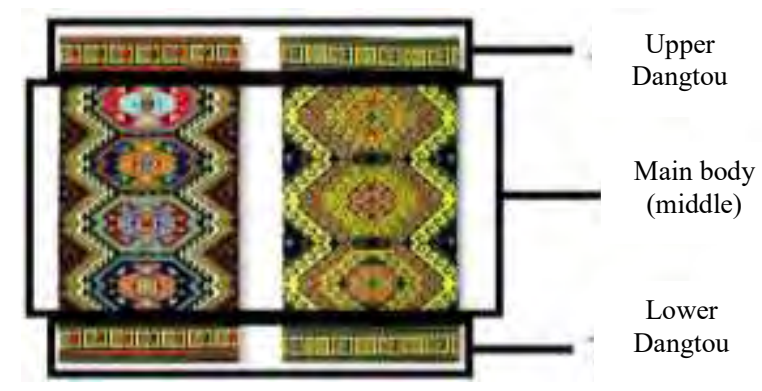

Fig. 2. Example of the basic composition of Tujia brocade.

2) Characteristics of typical pattern: Tujia brocade pattern not only shows the unique Tujia culture and customs, but also has many kinds, which can be roughly divided into the following categories:

a) Animal birds and beasts: The brocade pattern of this kind is relatively rich, not only takes a romantic style to highlight the characteristics of an animal, but also has many changes in form. And the two-dimensional expression method is used in the pattern, which makes the brocade pattern more vivid, highlights the characteristics of the pattern to be expressed, and shows the artistic characteristics of the brocade. Animal patterns often seen in brocade patterns are "phoenix pattern", "sparrow pattern and "Mabi Flower" and so on, which all convey the meaning of auspicious happiness. b) Flowers and plants: Flowers and plants pattern is a kind of Tujia brocade pattern after the ending of fishing and hunting life of Tujia people, so it appears later than animal birds and beasts pattern. Tujia people love and respect nature, and have unique feelings for all kinds of plants that exist in nature. In the brocades, they often incorporate yam, plum blossom, peony and other plants into the patterns, which not only convey the composition and structure of plant patterns, but also show Tujia people's cherishing of nature.

c) Geometrical hook pattern: Geometrical hook pattern is the most typical in Tujia brocade pattern. This kind of pattern usually takes eight hooks as the center, expanding to the surrounding layers, and then forms overlapping and interdependent hook pattern by using deep and light colors. For this kind of pattern, there is not only the pattern that many geometric patterns intersecting each other to form a whole, but also the pattern that only one geometric pattern can express the content. By splitting and combining these geometric elements of points, lines and surfaces, and then abstracting them, the novel, abstract and plain geometric patterns can be made. For example, diamond pattern, ten thousand-character pattern, double octagon pattern and so on can all express the abstract beauty.

d) Articles for daily use: In the eyes of the Tujia people, everything that one can see in the world can be called "flowers". There is beauty lies no matter in animal and flowers, or in living utensils. Tujia women can incorporate such beauty in the design of Tujia brocade patterns, such as "windows", "bamboo racks", "tables and chairs" and so on. The "chair flower" is one of the most representative brocade patterns of Tujia nationality. The prototype of this pattern is the chair in daily life. With the artificial extraction characteristics of Tujia weavers, the geometric and abstract patterns are designed. And the final "chair flower" pattern is formed according to the structure of the brocade.

\section{B. Color}

Tujia people emphasize the high purity in the use of color, pursuing the matching techniques of color contrast, for example, the use of complementary color like yellow with purple, red with green, orange with blue is very rare. In color matching, Tujia people often choose colors according to the background color. They usually use dark blue and black as the background color to highlight the strong and bright of other colors. The color of the main pattern is bright and gorgeous, the auxiliary pattern is mild, and the edge part is often transited by light color such as white or gray, which makes the overall pattern with clear distinction between primary and secondary, and the brocade bright and pleasant, vivid and generous.

\section{CUltural CONNOTATIONS}

The traditional brocade pattern of Tujia not only provides a lot of artistic aesthetic inspiration, but also demonstrates the distinct national character of Tujia from its cultural connotation. It is not enough to explore the ethnic art only from the aspect of representation characteristics, since a kind of folk art carries the ethnic history in a larger sense. Therefore, 
for the traditional Tujia brocade pattern, we need to make a thorough study from the belief, folk custom, daily life and the weaver himself.

\section{A. Nature Worship}

During the period of Tujia ancestors, because of the low social productivity, people depended on the natural forces that dominated their lives, so they personalized the natural forces into natural gods. Natural gods include not only natural objects, such as animals and plants, but also natural phenomena, such as clouds and sunshine, wind and rain, etc. In ancient China, the sun has always been regarded as the Supreme God by the ancestors, and the Tujia people worshiped the sun and ancestors. "Sun" is called "Laozi" in Tujia, which has the connotation of great gods. Tujia people worship and respect the sun, and make sacrifice to the sun. The sun rises from the east and falls to the west, shines on all things, brings light, warmth and infinite vitality to mankind. For Tujia ancestors, everything cannot be separated from the sun. The "sun pattern" in Tujia brocade pattern shows Tujia ancestors' respect for the sun god, and this natural worship more directly expresses their idea of respecting heaven.

\section{B. Totem Belief}

Totem is a kind of behavior that primitive ancestors worshipped animals, plants or non-living things. According to the primitive religious belief, totem belief was an earlier belief concept, while totem and totem worship produced in the development of primitive natural belief concept was also a special folk culture. As legend said, Tujia people are the descendants of the white tiger. Historically, they have the clan names of "White Tiger Descendants". Tujia people worshiped the white tiger as Tujia totem and regarded the white tiger as their religious belief. Therefore, the white tiger totem is a common totem belief of Tujia people. There are two kinds of legends about white tiger in Tujia area, one is called walking white tiger, and the other is sitting white tiger. The sitting white tiger, sitting in the hall, majestic-looking, is to be respected; while the walking white tiger, breaking the door, ferocious, is to be driven. To "respect" and "drive" white tigers were formed according to legends left by the ancestors.

The inheritance and development of Tujia brocade not only integrates Tujia people's aesthetic ideas, cultural customs and religious beliefs, but also condenses Tujia people's overall wisdom and has distinct Tujia characteristics. Not only are Tujia people satisfied with this, but also they are accepted by the people.

\section{REDESIGN OF TUJIA BROCADE PATtern}

With the continuous development of cultural pluralism, design has not only become a professional technology, but also an art to convey information and exchange emotions that connects different countries and different nationalities. From this paper we know that Tujia brocade pattern has symbolic, abstract and two-dimensional characteristics, which has certain coincidence with flat design concept and deconstruction design. Therefore, the concept of flat design and deconstruction design can be applied to the redesign of Tujia brocade pattern. The following is a case study of Tujia brocade pattern, Mabi Flower.

\section{A. Flattening Design of Pattern Deconstruction}

Because of the fast-paced modern life, people are more and more aware of the importance of information, accurate transmission of information has become an irreplaceable part of modern life, which gave birth to the concept of flattening design. The concept of flattening design emphasizes that elements should be simplified, abstracted and symbolized so that information can be expressed more intuitively and concisely.

In the format design, the flattening design generally adopts the method of "color block segmentation" or "mesh segmentation", dividing and arranging the surface with the equal ratio, which is both intuitive and neat. In the use of color, although emphases on color contrast, it generally does not use bright and strong color and tend to color with appropriate brightness. When using color, it often displays in the form of color combination. There is no gradual change between different color blocks. This simple form makes color design both with aesthetic in different level and the visual impact caused by conflict and contrast. In addition, white space is also a distinct feature of flattening design.

Deconstructionism is a derivative of structuralism, which rose in the 1970s and was widely disseminated in the 1980s. It is a very representative artistic form derived from the development of post-modern society. It inherits critically the past modernist design ideas and uses unique methods to break and reconstruct the existing elements, so as to produce a new concept. In the aspect of pattern design, deconstructionism attaches great importance to the original pattern itself, in which the design is to boldly reform the structure of the original pattern, that is, to split and reorganize to form a new structure.

Tujia brocade pattern is formed by two-dimensional creation of three-dimensional objects in nature and social activities. The process of simplifying natural objects and making plane description conforms to the process of flattening design. When deconstructing the design of Tujia brocade pattern, we should not only extract the "shape" of Tujia brocade pattern, but also need to pay attention to the organic coordination with its "meaning". With the in-depth understanding to the feelings of Tujia people in creating Tujia brocade, we can integrate it with modern aesthetic concepts, extract and refine traditional brocade patterns, and design new modern patterns.

"Mabi Flower" belongs to the animal pattern in Tujia brocade pattern. The main components of this pattern are: ponies, the sun and so on. The mouth, body and limbs of the pony are appropriately generalized into geometric shapes by straight lines and diagonals, and the main characteristics of the pony's movement are grasped by this highly generalized and abstract method. The "sun" in the design is a common "sunflower" pattern, in addition to showing the real scene of horses running under the sun, there is a meaning of worshiping the sun. So this pattern also reflects the worship of the sun by Tujia ancestors. In retaining the weaving structure of Tujia brocade, the two main patterns of "pony" and "sun" are 
redesigned according to the concept of flattening design, as shown in "Fig. 3" and "Fig. 4".

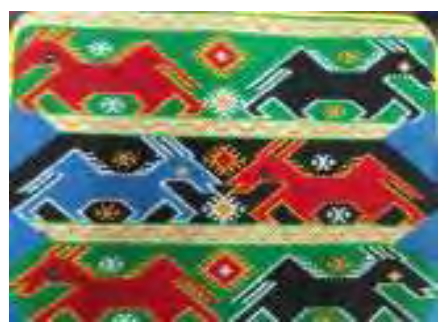

Fig. 3. "Mabi Flower" brocade pattern.
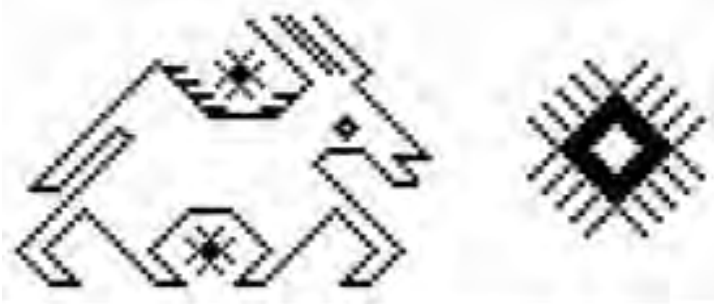

Fig. 4. "Mabi Flower" flattening design.

\section{B. Pattern Color Design}

Nature is the main source of inspiration for Tujia brocade art. Tujia people like colorful flowers and trees and all kinds of feathers. Therefore, all original bright and bold colors, such as yellow, red and blue, appear frequently in Tujia brocade, which not only conveys Tujia people's respect for nature and life, but also contains their expectations for the future. When expressing different emotions, we can classify the typical patterns in brocade patterns, analyze and extract the matching colors in different situations, and then apply them in different situations.

For example, the pattern of "Mabi Flower" has strong and vivid color matching, with rough, simple, generous and gorgeous ethnic style. A large amount of red and green, yellow and purple, blue and orange are contrasted in color, brightness and cold and warm respectively. The strong impact of contrasting color is alleviated by black (dark) warp yarn or light yellow lacing (Tujia people have the tradition of black and white taboo). The extraction of its color is conducive to the color harmony and unity of the subsequent creative design, as shown in "Fig. 5" and "Fig. 6".
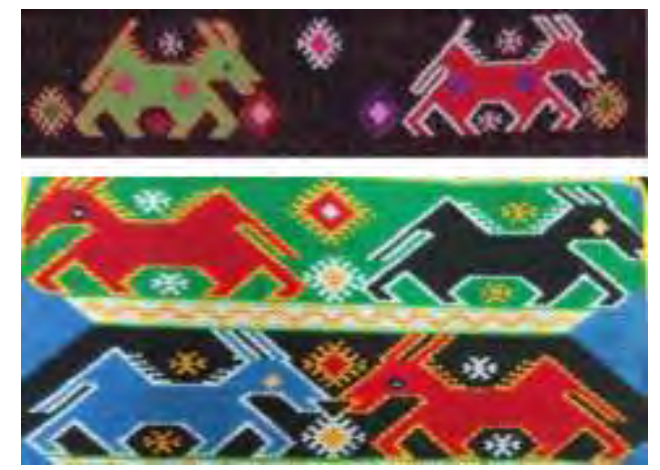

Fig. 5. "Mabi Flower" pattern color.

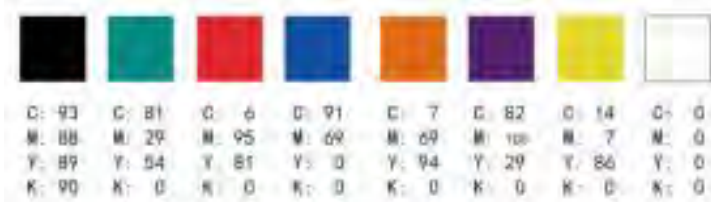

Fig. 6. "Mabi Flower" pattern color design.

According to the above research, the design concept of flattening and deconstruction correspond to the artistic characteristics of Tujia brocade pattern to a certain extent. Integrated the above two together, the charm of Tujia traditional culture is expressed by modern design method, and the brocade pattern and brocade culture of Tujia are better interpreted.

\section{THE APPLICATION IN MODERN CULTURAL AND CREATIVE DESIGN}

The emergence of the concept of cultural and creative design aims at the protection and inheritance of local culture. The incorporation of Tujia brocade patterns into the cultural and creative design is to publicize the ethnic minority spirit and spread the culture. On the basis of the extraction of pattern elements with purpose, and according to the needs of cultural creation design, we can employ Tujia brocade pattern elements in modern cultural creation design scientifically, adopting the way of turning the whole into zero, and through appropriate blank, unit form repetition and other ways, so that the creative design can display the modern and fashion aspect of the product. In the following part, we are trying to apply the flattening designed "Mabi Flower" patter into ornaments, scarves, bags, etc.

\section{A. The Application in Ornaments}

Ornaments refer to those worn on the head, such as earrings, necklaces, etc. Besides the function of decorating the human body, it also has the significance of showing social status, wealth and identity. The most primitive ornaments of mankind can probably be traced back to the remote Stone Age. Since the beginning of mankind's awareness of decoration and beautification of their own time, human beings and it has formed an indissoluble bond.

With the progress and change of the times, people's aesthetic concept tends to be more individually, and the aesthetic of decorations also presents diversified individual needs. Nowadays, with the continuous renewal of design concepts and the combination of traditional patterns and modern design, designers are given more freedom in imagination and design means, making each work both special and has inner beauty. Applying the flattening Tujia brocade pattern into modern ornaments meets modern people's pursuit of individualized aesthetics. The Tujia brocade pattern with Oriental national characteristics is integrated into the design of modern jewelry, and the deep exploration of innovative design of Tujia brocade pattern in modern jewelry is carried out. The jewelry with Chinese traditional artistic style is expected to show a new style. 
The "sunflower" elements of the flattened "Mabi flower" brocade pattern in the above is selected as the main component of the ornament design. The design is based on different sizes, shapes and other characteristics. The necklace design is taken as an example. In the necklace design, the two basic forms of "sunflower" elements are overlapped and staggered to form a new element, and then the two elements are arranged repeatedly and continuously to fit the appearance characteristics of the neck belt. Then the "pony" elements in "Mabi flower" are made into metal pendant and suspended in the middle of the necklace, as shown in "Fig. 7" and "Fig. 8".

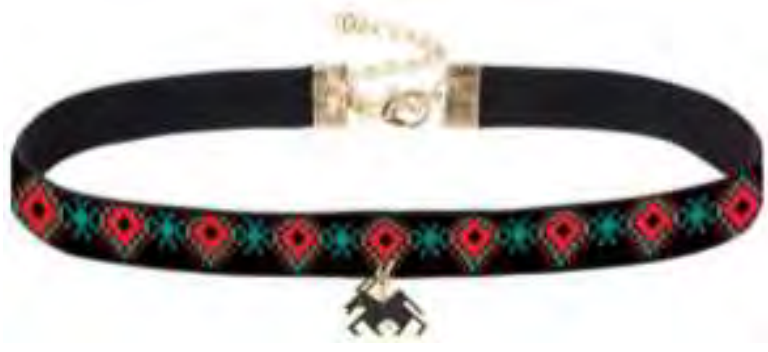

Fig. 7. "Mabi Flower" necklace design.

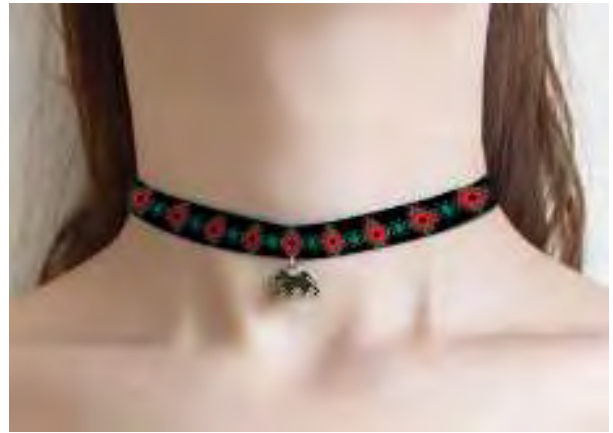

Fig. 8. "Mabi Flower" necklace design sketch.

The combination of Tujia brocade pattern and ornament makes ornament not only a practical product, but also an art with rich cultural connotations.

\section{B. The Application in Scarves}

Scares, as accessories of clothing, are the shining part of clothing. Nowadays, the use of scarves is not limited to the neck, but for more decorative areas, as waist belt; tied to the bag as a handbag decoration; hanging on the wall as a unique style decorative painting. In the innovative design of scarves, we cannot simply imitate and use other people's design rigidly. Instead, we should add formal beauty principles and design techniques to the traditional pattern and reconstruct the pattern composition, so as to meet the needs and aesthetic levels of our modern people.

Using the means of orderly placing, overlap, staggering, repetition and rotation, etc. to arrange the above flattened "Mabi Flower", and arranges the pattern in a balanced twoway continuous pattern composition. The edges and corners of scarves are complementary to each other. They are not only a part of the main body, but also a prominent point that attracts people's attention. Although the form and color of the pattern need to be unified, the successful corner design acts as a foil just like the frame with a picture. The size of the corner can be large or small, it can be a thin line, and can also be a wide edge composed of patterns. Such scarf design not only has the ethnic characteristics, but also in line with the trend of the times, and also be practical in people's daily life, as shown in "Fig. 9" and "Fig. 10".

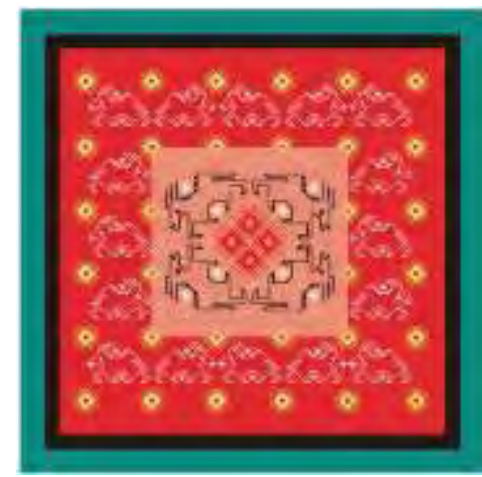

Fig. 9. "Mabi Flower" scarf pattern I.

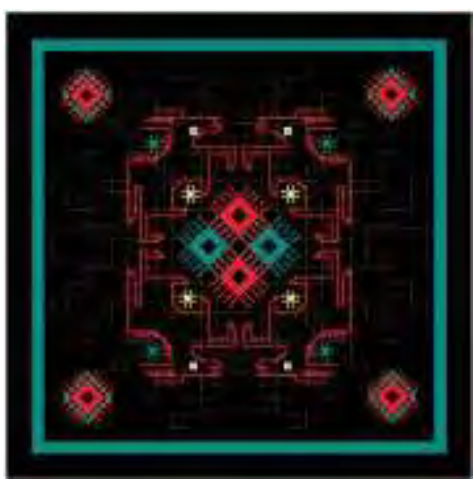

Fig. 10. "Mabi Flower" scarf pattern II.

Formal beauty in innovation is evolved from primitive form. On the basis of maintaining the basic elements of Tujia brocade pattern, it creates contemporary works of art by means of transformation, refining and innovation. And then it embodies the aesthetic design features of scattering and restructuring, and is not subject to the specific restrictions of pattern order and arrangement. It makes the Tujia culture being inherited and developed in the real sense, and thus creating a fashionable, artistic and contemporary fine scarf pattern.

\section{The Application in Bags}

Bags are not only used to store personal goods, but also reflect a person's identity, status, economic status and even personality. A practical bag that is fashion and has special design can add beauty to people. In addition to the innovation in the shape and materials, the design of pattern on the bag is also an important part in the design. If we want to design a bag pattern that can reflect the spiritual connotation of traditional culture, we should not use the traditional pattern directly, but make some changes and innovation according to the aesthetic needs of modern people. 
The design of bag pattern can be different according to different styles. Similarly, we use the flattened "Mabi Flower" pattern mentioned above as the basic element. The basic features of the two bags are elegant leather rectangular ladies' bag and lively wooden rectangular ladies' messenger bags. When we design the former, we should grasp the three key points of elegance, leather and rectangle, while the latter is closely linked to the three key points of liveliness, wood and rectangle. As shown in the following pictures, the starting point of our design must be different according to different needs. The overall design of the former is symmetrical, with black as the main color, conveying a sense of stable and mysterious, giving people a feeling of elegance; while the overall design of the latter is simple and easy, creating a sense of picture, making people feel comfortable and fresh. The edges and corners of scarves are complementary to the main part. They are not only a part of the main scarves, but also a prominent point that attracts people's attention. Although the form and color of the pattern need to be unified, the successful corner design acts as a foil just like the frame with a picture. The size of the corner can be large or small, it can be a thin line, and can also be a wide edge composed of patterns. Such scarf design not only has the ethnic characteristics, but also in line with the trend of the times, and also be practical in people's daily life., as shown in "Fig. 11" and "Fig. 12".

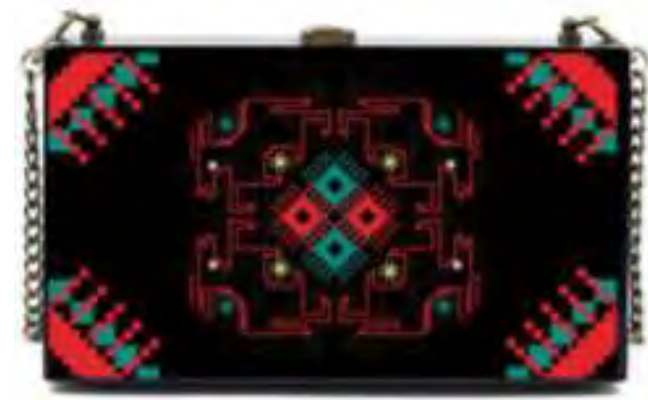

Fig. 11. "Mabi Flower" bag pattern I.

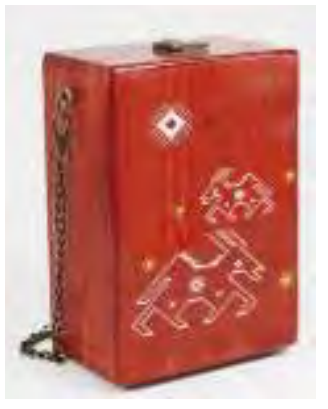

Fig. 12. "Mabi Flower" bag pattern II

At present, Tujia brocade technology has been inherited and promoted to a certain extent, but if we want to broaden the recognition scope of Tujia brocade, we must make the creative design with Tujia traditional elements meet the aesthetic and emotional needs of modern people, let the creative products spread Tujia's unique artistic charm, and realize the new development of Tujia brocade pattern.

\section{CONCLUSION}

With the collision and blending of design ideas in different countries, it is worth thinking about how to protect and inherit Tujia culture and decorative expression to make it shine in modern art design. This study finds that on the basis of understanding its material, technical and spiritual cultural background and connotation, it is an effective way to analyze the details of decorative patterns and to extract, deconstruct and reorganize them with modern design techniques. It is hoped that through the proposed design ideas, more people will be called on to pay attention to local culture and create designs with local style and in line with modern aesthetic concepts. In this way, we can not only promote the development of modern Chinese design, but also promote and disseminate traditional Chinese culture.

\section{REFERENCES}

[1] Liu Lian. Study on Aesthetic Characteristics and Application of Tujia Brocade Patterns in Western Hunan [D]. Hunan University of Science \& Technology, 2013, P14. (in Chinese)

[2] Zhu Yuting, Qian Lin. On the Elements of Flattening Design [J]. Literature - Theory, 2014. 11, P262. (in Chinese)

[3] Peng Yi. Tujia Brocade Pattern and Its Application in Modern Design [D]. Hunan Normal University, 2010, P27. (in Chinese)

[4] Fu Huan. Design Style in Interface Design — Flattening Design and Quasi-Physical Design [J]. Literature · Literature Art, 2013. 8, P180. (in Chinese)

[5] Han Yuting, Han Fan. Study on the Application of Tujia Brocade Patterns in Modern Design in Western Hunan [J]. Guizhou Ethnic Studies, 2015. 08, P99. (in Chinese)

[6] Huang Baiquan, You Hongbo. The Development and Evolution of Tujia Brocade and Its Modern Enlightenment [J]. Journal of Hubei Institute for Nationalities, 2005.23, P8. (in Chinese) 